\title{
A new meta-module for efficient reconfiguration of hinged-units modular robots*
}

\author{
Irene Parada ${ }^{1}$, Vera Sacristán ${ }^{2}$, and Rodrigo I. Silveira ${ }^{2}$
}

\begin{abstract}
We present a robust and compact meta-module for edge-hinged modular robot units such as M-TRAN, SuperBot, SMORES, UBot, PolyBot and CKBot, as well as for centralpoint-hinged ones such as Molecubes and Roombots. Thanks to the rotational degrees of freedom of these units, the novel meta-module is able to expand and contract, as to double/halve its length in each dimension. Moreover, for a large class of edge-hinged robots the proposed meta-module also performs the scrunch/relax and transfer operations required by any tunneling-based reconfiguration strategy, such as those designed for Crystalline and Telecube robots. These results make it possible to apply efficient geometric reconfiguration algorithms to this type of robots. We prove the size of this new meta-module to be optimal. Its robustness and performance substantially improve over previous results.
\end{abstract}

\section{INTRODUCTION}

Modular self-reconfigurable robots are connected sets of units that can change their connectivity, varying the shape of the robot. Thus, these systems can modify their morphology (reconfigure) to better suit different tasks and environments and to self-repair. This makes them more versatile and robust than fixed-shape unique-purpose robots.

They can be classified according to different criteria: architecture and topology, connections, degrees of freedom, propulsion method, etc. From the geometric viewpoint, one very interesting class is that of all robots whose units are able to expand and contract, since this property can be exploited by reconfiguration algorithms. As shown in the top row of Fig. 1, this kind of unit allows movements interior to the robot configuration, leading to tunneling reconfiguration strategies, in which modules travel through the volume of the robot. The bottom row of Fig. 1 shows how the same reconfiguration is achieved by means of a surface strategy, i.e., by moving the units along the boundary of the configuration.

The tunneling capability is particularly interesting because it can be exploited to reconfigure robotic systems in-placeusing only the space occupied by the source and target configurations - with a small number of moves and parallel steps. Moreover, as the volume of a robot configuration grows, there is proportionally less surface area per module.

*This work was partially supported by Projects MTM2012-30951 and Gen. Cat. DGR 2014SGR46. R. I. Silveira was also partially supported by MINECO through the Ramn y Cajal program and I. Parada was supported by the Austrian Science Fund (FWF): W1230, Doctoral Program "Discrete Mathematics".

${ }^{1}$ Irene Parada is with the Institute of Software Technology, Graz University of Technology, Austria iparadalist.tugraz.at

${ }^{2}$ Vera Sacristán and Rodrigo I. Silveira are with the Departament de Matemàtica Aplicada II, Universitat Politècnica de Catalunya, Barcelona, Spain $\{$ vera.sacristan, rodrigo.silveira\}@upc.edu

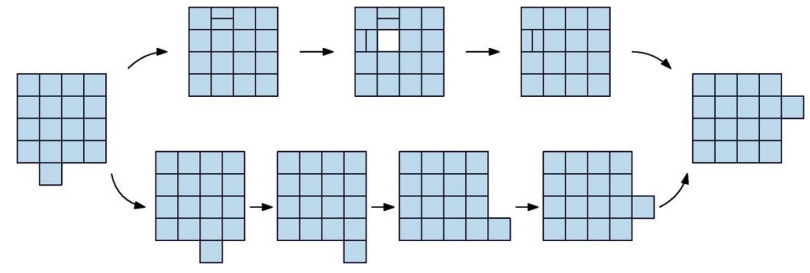

Fig. 1. Different reconfiguration strategies. Top: tunneling algorithm for expandable and contractible modules. Bottom: surface algorithm.

This is an impediment to parallelism in surface strategies, causing the reconfiguration speed to decrease as the number of modules increases.

Physical prototypes of self-reconfiguring systems with square or cubic units that can expand and contract by a factor of two in each of its dimensions are Crystalline robots [18] in two dimensions and Telecubes [21] in three dimensions. Other modular robots incorporating the concept of expansion and contraction include the Metamorphic robot [14], whose 2-dimensional hexagonal pieces are able to modify their angles; PolyPod [23], a chain and bipartite robot with 2-DOF contractible segments; TETROBOT [7], a truss-structured robot composed of rigid or actuated struts and joints allowing rotation; and Odin [11], a versatile 3-dimensional robot which uses telescopic links.

Several tunneling algorithms for universal reconfiguration have been proposed for Crystalline and Telecube robots. In all of them the units are grouped into meta-modules of at least $2 \times 2(\times 2)$ units. The melt-grow [18] is a centralized algorithm which reconfigures any connected robot of $n$ units in $O\left(n^{2}\right)$ moves and steps. The Pac-Man algorithm [5] and the algorithm in [22] use $O\left(n^{2}\right)$ parallel steps.

In-place reconfiguration, in which the space in which the modules can move is restricted to the union of the source and target configurations, is also possible. Maintaining the assumptions of constant velocity and strength, under which a module can pull or push only a constant number of other modules at constant speed, $2 \times 2(\times 2)$-unit meta-modules can be used to reconfigure in-place. This can be achieved by both centralized [2] and distributed [16] algorithms, and the overall number of unit moves needed is $\Theta\left(n^{2}\right)$, which is optimal in this setting. If modules have linear strength, the total number of unit moves can be reduced to $O(n)$ [3]. With this strength requirements and allowing velocities to build up over time, reconfiguration is possible in $O(\log n)$ parallel steps and $O(n \log n)$ overall moves [4].

Many current modular robot prototypes have other very 

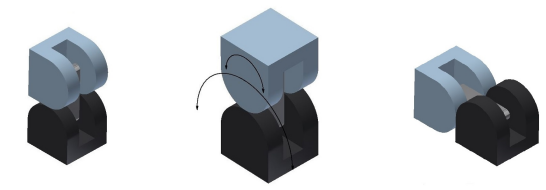

Fig. 2. An edge-hinged unit and its degrees of freedom, as they appear in M-TRAN.

convenient properties such as chain/tree or hybrid architectures, high mobility of their units or locomotion capabilities, but cannot expand and contract. In this paper, we show how it is possible to apply the previously described algorithms also to some of these robots by constructing meta-modules with their units, which cannot expand and contract, such that the whole meta-module can.

Due to the interest of the tunneling capabilities, metamodules of other robots have been designed that are able to perform the expand and contract operations. For example, Kotay and Rus [9] have proposed an expandable and contractible meta-module for Molecules, while Murata and Kurokawa have presented in [12] a small and compact MTRAN meta-module, but it can only expand and contract in two dimensions. For 3D, the only expanding/contracting MTRAN meta-module that we are aware of is that of Aloupis et al. [1], which is also valid for Molecube [26]. However, the meta-module of [1] is formed by 58 units and the side length of its minimum axis-aligned bounding cube when expanded is 32 units. In addition, it is much less compact than the one by Murata and Kurokawa, making it less robust.

The interest in the M-TRAN series of modular robots, from M-TRAN I to M-TRAN III [10], is probably due to their simplicity and, at the same time, their versatility. MTRAN is one of the geometrically simplest examples of a large class of edge-hinged robots from the viewpoint of its degrees of freedom. Its units, depicted in Fig. 2, can be connected in a chain or tree topology which allows for continuous movement. This makes M-TRAN suitable for a variety of tasks and locomotion modes: it can perform whole body motions as a crawler or as a travelling wave, as well as several legged gaits [12]. At the same time, the blocks constituting M-TRAN units can be arranged in a cubic grid. In this lattice architecture, reconfiguration is simpler to plan. Something similar happens with central-point-hinged modular robots such as Molecubes or Roombots [20], even though their hinging mechanism gives rise to a geometrically different rotation movement of their units.

M-TRAN and, more generally, most of edge-hinged modular robot units consist of two edge-linked semi-cylindrical cubes, as illustrated in Fig. 2. We refer to these semicylindrical cubes as blocks. Each block has a gender (male/female) and connectors (different for the two genders) on its three flat surfaces. Two units can be attached by flat surfaces of different gender, in any of the four possible relative orientations. The units have two degrees of freedom: each semi-cylindrical block can rotate from $-90^{\circ}$ to $90^{\circ}$ with respect to the link joining both blocks.
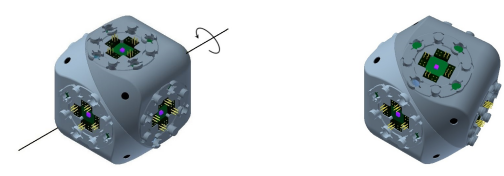

Fig. 3. A central-point-hinged unit and its rotational degree of freedom, as it appear in Molecubes. Model from the Molecubes project [26].

In contrast, Molecubes [26], as an example of centralpoint-hinged units, are cube-shaped with two halves that can rotate relative to each other. As we can see in Fig. 3, the rotation axis passes through the center of the cube and through two opposite vertices. All faces of a Molecube unit can be actively connected in any possible orientation.

\section{A. Contributions}

In Section II we present a versatile and more realistic meta-module, smaller in both size and number of modules, for edge-hinged modular robots that is able to perform the expand/contract operations of Crystalline and Telecube units. In particular, the meta-module we propose is valid for MTRAN, but also for SuperBot [19], SMORES [6] UBot [25], PolyBot (G3) [24] and CKBot [15], which are edge-hinged modular robots. In Section III this result is extended to also deal with central-point-hinged modular robots such as Molecubes and Roombots. Moreover, since the metamodules of expandable and contractible units required by the reconfiguration algorithms would lead to meta-meta-modules of our edge-hinged units, in Section IV we show that for most of the edge-hinged robots these meta-meta-modules are not necessary if our meta-module is used. As a side effect, we obtain that our meta-module can also be used in surface traversing reconfiguration strategies based on the sliding cube model.

\section{A SMALLER AND MORE ROBUST META-MODULE}

In this section we describe how both kinds of units (edgehinged and central-point-hinged) can be combined into a meta-module that is able to expand and contract. We will prove its correctness for M-TRAN, which implies it for SuperBot, SMORES, UBot, PolyBot, and CKBot. In the next section we will prove it for for Molecubes, which implies it for Roombots.

The proposed meta-module for M-TRAN (in general, edge-hinged) units, illustrated in Fig. 4, consists of 6 arms, aligned in three directions that are parallel to the $x, y$ and $z$ axes. Each arm is implemented using a 2-unit chain: two units attached at square flat faces and with the direction of their links aligned, as shown in Fig. 5. The key property of these arms is that the rotation of the blocks within the units allows them to contract an expand, while preserving potential connections.

Lemma 1: The M-TRAN arm can be contracted. During this operation its two extremal blocks stay aligned and keep their orientation.

Proof: The contraction operation is shown in Fig. 5. Its realization is allowed by the two rotational degrees of 
freedom and the semi-cylindrical shape of the blocks. It is easy to see that this operation does not change neither alignment nor the orientation of the extremal blocks of the chain.

The pairs of arms of the M-TRAN meta-module that are oriented in the same direction are connected to each other, resulting in a 4-unit chain whose blocks are all aligned. However, the linkages of the two connected arms differ in their orientation (see Fig. 6 left). We call the blocks connecting the two arms central.

The six arms of the M-TRAN meta-module form three 4unit chains, one for each of the $x, y$ and $z$ directions, attached through their central blocks at their semicircular faces. Fig. 4 shows the attachment of the central blocks (center) and the meta-module with all its arms expanded (left) and all its arms compressed (right).

The meta-module can contract or expand each arm independently while keeping the six central blocks still. Since the linkages of the two arms forming a 4-unit chain have different directions, their contraction and expansion movement takes place on two orthogonal planes, as illustrated in Fig. 6 right.

Lemma 2: No self-intersection is produced when expanding or contracting any arms of the M-TRAN meta-module.

Proof: Consider the minimum axis-aligned cube containing the expanded meta-module and decompose it into eight octants. It is easy to see that each expanded arm is contained in a different octant. The plane on which the contraction of an arm occurs always has a region in the corresponding octant (see Fig. 7). Therefore, when we contract an arm, we can always use the octant that is exclusive to that arm. This guarantees that collisions cannot occur.

Lemma 3: During the expansion and contraction of any subset of arms of a M-TRAN meta-module the robot stays connected.

Proof: While expanding and contracting any single arm, the central blocks remain immobile. These six blocks
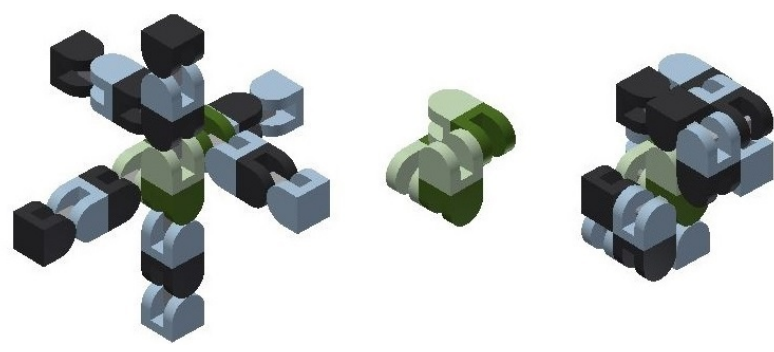

Fig. 4. Meta-module for edge-hinged units. Left: all arms expanded. Center: central blocks. Right: all arms contracted.
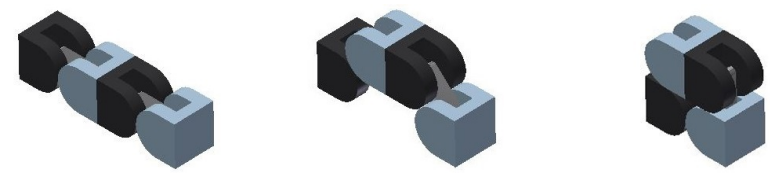

Fig. 5. The M-TRAN arm is able to contract while maintaining its potential connections at both ends.

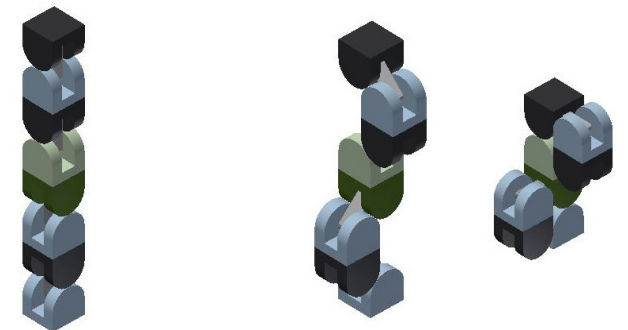

Fig. 6. Left: connecting two M-TRAN arms into a 4-unit chain. The central blocks are highlighted in green. Right: the compression movement of one 4-unit chain.

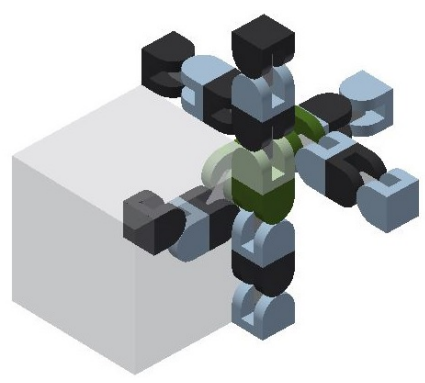

Fig. 7. M-TRAN meta-module contracting an arm in its corresponding octant.

maintain the meta-module connected at all times. Moreover, connectivity with neighboring meta-modules is preserved: if the tip of an arm is attached to the tip of another metamodule arm, Lemma 1 guarantees that this attachment can be maintained during expansion and contraction.

Theorem 4: The M-TRAN meta-module can perform the Crystalline and Telecube unit operations: expand, contract, attach, and detach.

Proof: From the previous lemmas we conclude that the length of the meta-module can be reduced by half (when expanded arms are contracted) or doubled (when contracted arms are expanded) in any of the $x, y$ and $z$ directions. This can be done while preserving connectivity (Lemma 3 ) and avoiding collisions (Lemma 2).

Corollary 5: Theorem 4 is also valid if the meta-module is made out of Superbot, SMORES, UBot, PolyBot(G3), and CKBot robot units.

Proof: The result is valid for Superbot units, since they can perform M-TRAN moves. It applies to SMORES because two SMORES units can behave like one Superbot unit [6]. Finally, it is also valid for UBot, PolyBot(G3), and CKBot (using self-reconfigurable connectors), since four units of any of these robots can behave as one M-TRAN 2unit chain. However, the meta-module is not valid for iMobot units [17] since the bounding box of their semi-cylindrical blocks is a right rectangular prism but not a cube.

Consider a lattice such that in the expanded configuration of the meta-module each of the M-TRAN blocks fits in a unit cell. We call size of the meta-module the length of its minimum bounding cube. In other words, the size of the meta-module measures the resolution of any configuration of meta-modules. 
Theorem 6: The M-TRAN meta-module has optimal size.

Proof: Due to space limitations, the proof is only sketched. Since the meta-module must be able to contract to fit in a cube of half side length, the size has to be even. As the size of our meta-module is 8 , the remaining options are 2 , 4 and 6. Since one M-TRAN unit fits in two lattice cells, the size cannot be 2 . Size 6 would imply that the bounding cube in the contracted configuration has side length 3 . However, the 3D checker board property holds for M-TRAN units: if we color our lattice with two alternating colors, any configuration with each block fitting in a cell has male and female blocks in cells of different color. In a cube of odd length, cells on opposite faces have the same color. Thus, there is no possible connected configuration of contracted meta-modules of size 6 . Finally, size 4 can be discarded using integer programming techniques: guaranteeing connectivity requires more modules than can fit in one $2 \times 2 \times 2$ bounding cube in the contracted configuration.

\section{EXTENSION TO THE CENTRAL-POINT-HINGED CASE}

The meta-module for central-point-hinged units like Molecubes and Roombots is similar to the one described in Section II when expanded, as it also consists of 6 arms aligned in the $x, y$ and $z$ directions (see Fig. 8). In this case, though, the expansion/contraction does not occur on a plane (see Fig. 9). Therefore the shapes of the two modules differ when contracted and along the contraction.

The design of the meta-module carefully connects each arm through its central immobile half-block to the central immobile half-blocks of another two arms. In addition, arms are bent in different directions in order to avoid collisions.

In order to prove Lemma 1 for Molecubes, we follow the lemmata structure proposed in [1], whose proofs admit a straightforward adaptation to our design of the arm. Following the notation from [1], let $J_{i}$ and $O_{i}$ respectively denote the rotating half and the center of unit $U_{i}$, and let $x\left(O_{i}\right)$ denote the $x$-coordinate of $O_{i}$. We prove that during the expansion/contraction of an arm the following holds: $i$ ) the component connecting $J_{2}$ and $J_{3}$ is translated in parallel while maintaining the same orientation; $i$ i) $x\left(O_{1}\right) \leq$ $x\left(O_{3}\right)<x\left(O_{5}\right) \leq x\left(O_{7}\right)$; iii) the two extremal half-cubes of the arm stay aligned and keep their orientation; iv) the arm does not self-intersect.

In contrast to the two-dimensional contraction of the MTRAN arm, contracting and expanding the Molecube arm requires the use of the third dimension: one side for the rotating halves $J_{1}$ and $J_{4}$ and the other for some portions of $U_{2}$ and $U_{3}$ (see Fig. 9). This makes the proof of Lemma 2 more difficult in the Molecube case. Due to space constraints, we only sketch this proof. Divide the bounding box of the expanded Molecube meta-module into octants of identical size. Six of them contain one arm, and they are not intersected by any other arm during the contraction. By Lemma 1 (for Molecubes) no collisions occur in these octants. The other two octants, though, are intersected by the rotating halves $J_{1}$ and $J_{4}$ of two different arms and by part of $U_{2}$ and $U_{3}$ of a third arm during contraction. The rounded shape of the
Molecubes ensures that no collision occurs in neither of these two octants (note that if the cubes corner were not rounded, collisions would actually happen).

The analog of Lemma 3 for Molecubes follows from Lemma 1 and the fact that central half-blocks remain immobile during expansion/contraction and connect all arms together.

Therefore, Theorem 4 also holds for Molecubes. In fact, it also applies to Roombots, since one Roombot unit is geometrically equivalent to two connected Molecube units, with an extra rotational degree of freedom between them.

\section{AVOIDING META-META-MODULES}

By Theorem 4, we can apply the tunneling reconfiguration algorithms in [2], [5], [18], [22] for Crystalline and Telecube units to our meta-module. These algorithms, in turn, use meta-modules of Crystalline or Telecube units that are able to perform the following operations:

- Scrunch and Relax: compressing two neighboring connected meta-modules so that both occupy the same single lattice cell, and the reciprocal operation.

- Transfer: a compression in a meta-module is transferred to an adjacent lattice cell whose meta-module is not compressed.

In Section II we have shown that the new meta-module is able to perform the expand and contract Crystalline and Telecube unit operations. In this section we show that the M-TRAN meta-module is also able to perform the scrunch, relax, and transfer operations. This decreases the resolution of the configurations that are needed, both in size and number of units, since the reconfiguration algorithms can be applied without the need of meta-meta-modules of M-TRAN units and, more generally, of any analogously edge-hinged units like Superbot and SMORES.

In a scrunch operation one of the meta-modules stays still, guaranteeing the connectivity of the overall structure. The other meta-module adopts a position that we call canonical, which has the following properties:

1) The arms of the moving meta-module are parallel to those of the still meta-module, and they are all connected at their central blocks.

2) The symmetry of the resulting configuration allows to perform a relax operation on the moving meta-module to place it in any of the six adjacent lattice cells.

Fig. 10 (top) illustrates two adjacent meta-modules of edgehinged units before and after a scrunch/relax operation.

In a transfer operation two adjacent meta-modules stay still, while the other moves from the canonical position attached to one of the still meta-modules to the canonical position attached to the other. See Fig. 10 (bottom) for an illustration. The low density [13] of the configuration with two meta-modules in the same bounding box, as shown in Fig. 10, allows performing the scrunch/relax and transfer operations. Their actual implementation is rather involved. It comprises 49 independent moves of the six arms of the moving meta-module for the scrunch operation and 58 for the transfer operation. This leads to the following result. 

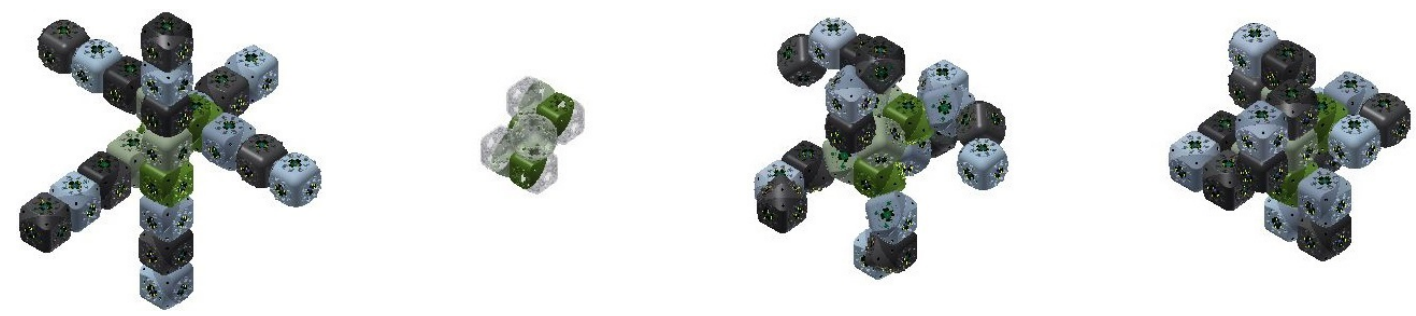

Fig. 8. Meta-module for central-point-hinged units. From left to right: expanded, center, contracting $\left(60^{\circ}\right)$, contracted.

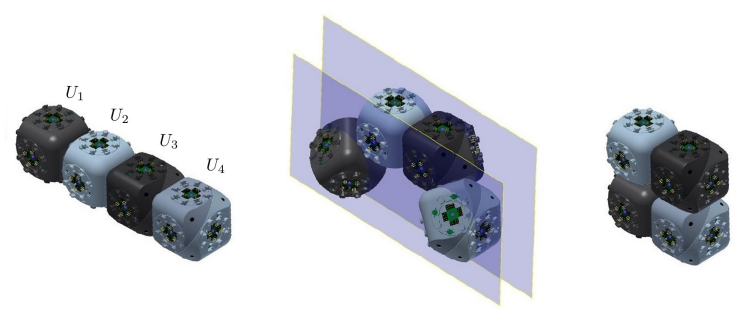

Fig. 9. The Molecube arm is also able to contract while maintaining its potential connections at both ends. During contraction the centers of the units do not stay in the same plane.

Theorem 7: The meta-module can perform the Crystalline and Telecube meta-module operations scrunch/relax and transfer.

Proof: Due to space constraints, only a sketch of the proof is provided.

First we show that both scrunch/relax and transfer can be obtained by only moving the scrunching, relaxing or transferred meta-module, while all the remaining elements of the robot configuration stay still. In other words, we prove that each 4-chain of the moving meta-module is able to move in all $x, y$ and $z$ directions as to reach its final destination as a whole, i.e., without ever disconnecting any of its units.

Connectivity of the entire robot is then guaranteed by the fact that every step (arm movement) keeps the corresponding 4-unit chain attached to the static modules of the configuration through some of its attachments.

We finally prove that no collision can occur when several operations are performed in parallel, since the moving arms never exceed the two adjacent bounding cubes shown in Fig. 10. When defining the moves, we have taken into account that one unit-size lattice cell can be temporarily exceeded by the corners of a prism-shaped half-block during a rotation. Consider, for example, the rotation transforming the central configuration of Fig. 2 into the right one. Notice that two lattice cells need to be empty in addition to the source, intermediate and target cells for the rotation to be performed without collisions. In order to avoid potential collisions like the one we just described, some steps of the scrunch/relax operation cannot be performed in a straightforward manner and require using carefully designed movements.

Visualizations of all the moves are provided in the video accompanying this paper.

\section{DISCUSSION}

We have presented a new meta-module for edge-hinged modular robots such as M-TRAN, Superbot, SMORES, UBot, PolyBot, and CKBot that can simulate the expanding and contracting properties of Crystalline and Telecube robots.

The use of meta-modules allows to greatly extend the functionality of modular robotic systems by mimicking other systems, at the cost of an increase in the number of modules needed. For this reason, meta-modules have received attention in the past (see [13], [9], [12], [1], [8]) and we believe that further enhancing their capabilities while keeping them small is still an interesting challenge, specially in the perspective of progressive miniaturization provided by nanotechnology. Moreover, the capability increase provided by meta-modules may be interesting in order to simplify the manufacture of modular robots units while keeping or even enhancing their versatility.

The novel meta-module is built with 12 robot units. When expanded, its size is 8 units and we have proven that this value is optimal. Thus, the number of units is significantly reduced with respect to the 58-units meta-module presented in [1] and its size is reduced to one quarter. It is also very compact if compared with the 54-Molecule meta-module [9]. We have extended this result to central-point-hinged modular robots, such as Molecubes and Roombots. Furthermore, robustness is also improved over the previous meta-module: when contracted, the new meta-module has only two corner joints per arm, as opposed to the four used in previous work, and leaves no gaps, making it much more compact. Therefore, the novel meta-module improves over previous results in the number of units required and the space used, and has a more compact and robust structure.

Moreover, it is the first time that it has been proved that tunneling algorithms, for which relevant complexity results exists, both in terms of time and space, can be applied to edge-hinged modular robots without requiring the use of meta-meta-modules. We hope that a similar reduction can be proved for other modular robotic systems, particularly for central-point-hinged modular robots.

Our result provides a general framework within which it applies. On the one hand, the new meta-module applies to a large class of edge-hinged modular robots. On the other hand, it is able to expand and contract, as well as to scrunch, relax and transfer. This implies that it can also perform slide and convex transition operations (by a concatenation of scrunchtransfer-relax moves) and, therefore, it can also be used in 


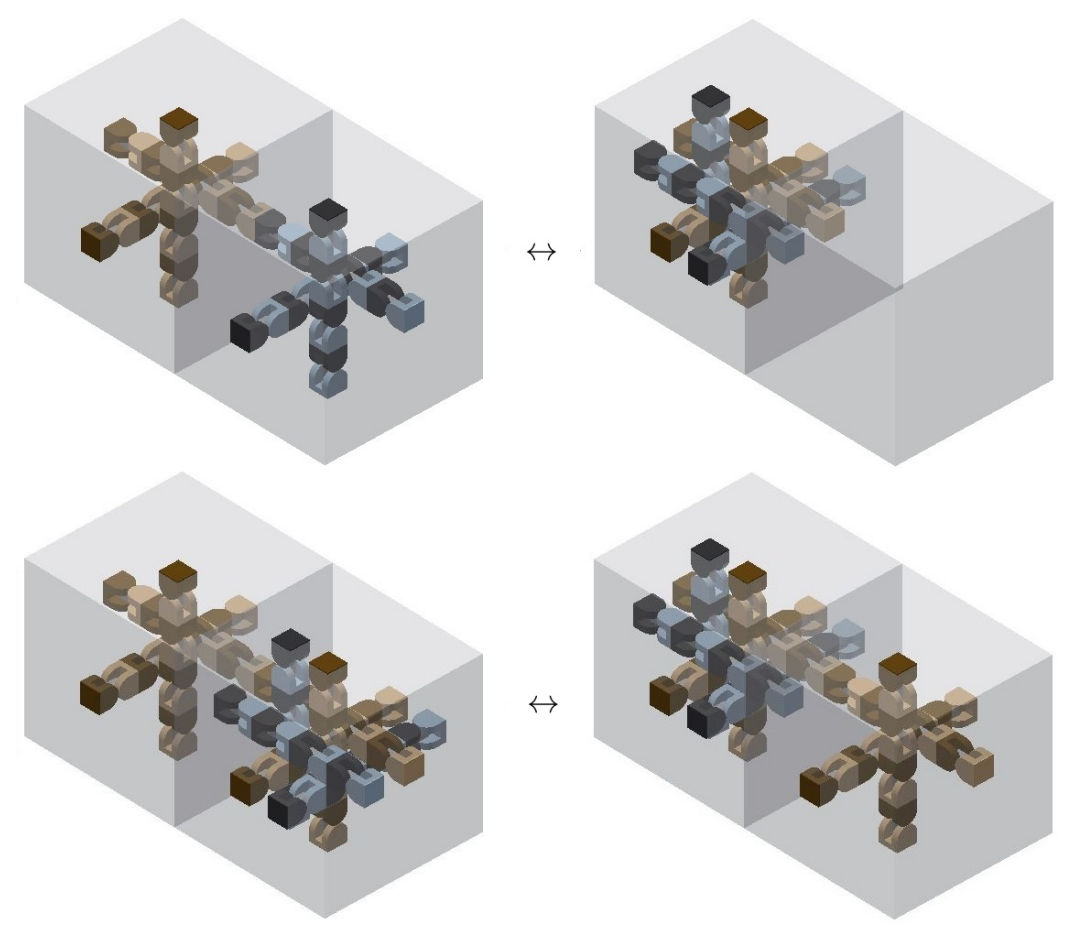

Fig. 10. Top: the scrunch/relax operation. Bottom: the transfer operation. Notice the canonical position of the blue meta-module when compressed.

reconfiguration algorithms based on surface strategies.

\section{REFERENCES}

[1] G. Aloupis, N. Benbernou, M. Damian, E.D. Demaine, R. Flatland, J. Iacono, and S. Wuhrer. Efficient reconfiguration of lattice-based modular robots. Comp. Geom.-Theor. Appl., 46(8):917-928, 2013.

[2] G. Aloupis, S. Collette, M. Damian, E.D. Demaine, R. Flatland, S. Langerman, J. O'Rourke, V. Pinciu, S. Ramaswami, V. Sacristán, and S. Wuhrer. Efficient constant-velocity reconfiguration of crystalline robots. Robotica, 29(1):59-71, 2011.

[3] G. Aloupis, S. Collette, M. Damian, E.D. Demaine, R. Flatland, S. Langerman, J. O'Rourke, S. Ramaswami, V. Sacristán, and S. Wuhrer. Linear reconfiguration of cube-style modular robots. Comp. Geom.-Theor. Appl., 42(6-7):652-663, 2009.

[4] G. Aloupis, S. Collette, E. D. Demaine, S. Langerman, V. Sacristán, and S. Wuhrer. Reconfiguration of cube-style modular robots using $O(\log n)$ parallel moves. In Proc. 19th Annual International Symposium on Algorithms and Computation (ISAAC), pp. 342-353, 2008.

[5] Z. Butler and D. Rus. Distributed planning and control for modular robots with unit-compressible modules. Int. J. Rob. Res., 22(9):699715, 2003.

[6] J. Davey, N. Kwok, and M. Yim. Emulating self-reconfigurable robots - design of the SMORES system. In Proc. IEEE/RSJ Int. Conf. Intell. Robot. Syst. (IROS), pp. 4464-4469, 2012.

[7] G. Hamlin and A. Sanderson. Tetrobot: A Modular Approach to Reconfigurable Parallel Robotics. Kluwer Academic Publishers, 1998.

[8] F. Hurtado, E. Molina, S. Ramaswami, and V. Sacristán. Distributed reconfiguration of 2D lattice-based modular robotic systems. Autonomous Robots 38(4):383-413, 2015.

[9] K.D. Kotay and D. Rus. Algorithms for self-reconfiguring molecule motion planning. In Proc. IEEE/RSJ Int. Conf. Intell. Robot. Syst. (IROS), pp. 2184-2193, 2000.

[10] H. Kurokawa, K. Tomita, A. Kamimura, S. Kokaji, T. Hasuo, and S. Murata. Distributed self-reconfiguration of M-TRAN III modular robotic system. Int. J. Rob. Res., 27(3-4):373-386, 2008.

[11] A. Lyder, R. Garcia, and K. Stoy. Mechanical design of Odin, an extendable heterogeneous deformable modular robot. In Proc. IEEE/RSJ Int. Conf. Intell. Robot. Syst. (IROS), pp. 883-888, 2008.

[12] S. Murata and H. Kurokawa. Self-Organizing Robots. Springer-Verlag, 2012.
[13] A. Nguyen, L. Guibas, and M. Yim. Controlled module density helps reconfiguration planning. In Proc. 4th Int. Workshop on Algorithmic Foundations of Robotics (WAFR), pp. 23-25, 2000.

[14] A. Pamecha, C.-J. Chiang, D. Stein, and G. Chirikjian. Design and implementation of metamorphic robots. In Proc. ASME Design Engineering Technical Conf. \& Computers in Engineering Conference (IDETC/CIE), pp. 18-22, 1996.

[15] M. Park and M. Yim. Distributed control and communication fault tolerance for the CKBot. In Proc. ASME/IFToMM Int. Conf. Reconfig. Mech. and Robots (ReMAR), pp. 682-688, 2009.

[16] M. Perera. Reconfiguración distribuida de robots cristalinos (Distributed reconfiguration of crystalline robots). Degree thesis under the supervision of V. Sacristán, Facultat d'Informàtica de Barcelona, Universitat Politècnica de Catalunya, 2015.

[17] G.G. Ryland and H.H. Cheng. Design of iMobot, an intelligent reconfigurable mobile robot with novel locomotion. In Proc. IEEE Int. Conf. Robot. Autom. (ICRA), pp. 60-65, 2010.

[18] D. Rus and M. Vona. Crystalline robots: Self-reconfiguration with compressible unit modules. Auton. Robots, 10(1):107-124, 2001.

[19] B. Salemi, M. Moll, and W.-M. Shen. Superbot: A deployable, multifunctional, and modular self-reconfigurable robotic system. In Proc. IEEE/RSJ Int. Conf. Intell. Robot. Syst. (IROS), pp. 3636-3641, 2006.

[20] A. Spröwitz, S. Pouya, S. Bonardi, J. van den Kieboom, R. Möckel, A. Billard, P. Dillenbourg, and A.J. Ijspeert. Roombots: reconfigurable robots for adaptive furniture. IEEE Comp. Int. Mag., 5(3):20-32, 2010.

[21] J.W. Suh, S.B. Homans, and M. Yim. Telecubes: Mechanical design of a module for self-reconfigurable robotics. In Proc. IEEE Int. Conf. Robot. Autom. (ICRA), pp. 4095-4101, 2002.

[22] S. Vassilvitskii, M. Yim, and J. Suh. A complete, local and parallel reconfiguration algorithm for cube style modular robots. In Proc. IEEE Int. Conf. Robot. Autom. (ICRA), pp. 117-122, 2002.

[23] M. Yim. New locomotion gaits. In Proc. IEEE Int. Conf. Robot. Autom. (ICRA), pp. 2508-2514, 1994.

[24] M. Yim, and Y. Zhang, K. Roufas, D. Duff, and C. Eldershaw. Connecting and disconnecting for chain self-reconfiguration with PolyBot. IEEE/ASME T. Mech., 7(4):442-451, 2002.

[25] J. Zhao, X. Cui, Y. Zhu, and S. Tang. UBot: a new reconfigurable modular robotic system with multimode locomotion ability. Industrial Robot 39(2):178-190, 2012.

[26] V. Zykov, A. Chan, and H. Lipson. Molecubes: An open-source modular robotics kit. In Workshop on Self-Reconfigurable Robotics at IROS, 2007. 